\title{
A NON-GAUSSIAN ORNSTEIN-UHLENBECK PROCESS FOR ELECTRICITY SPOT PRICE MODELING AND DERIVATIVES PRICING
}

\author{
FRED ESPEN BENTH, JAN KALLSEN, AND THILO MEYER-BRANDIS
}

\begin{abstract}
We propose a mean-reverting model for the spot price dynamics of electricity which includes seasonality of the prices and spikes. The dynamics is a sum of non-Gaussian Ornstein-Uhlenbeck processes with jump processes giving the normal variations and spike behaviour of the prices. The amplitude and frequency of jumps may be seasonally dependent. The proposed dynamics ensures that spot prices are positive, and that the dynamics is simple enough to allow for analytical pricing of electricity forward and futures contracts. Electricity forward and futures contracts have the distinctive feature of delivery over a period rather than at a fixed point in time, which leads to quite complicated expressions when using the more traditional multiplicative models for spot price dynamics. We demonstrate in a simulation example that the model seems to be sufficiently flexible to capture the observed dynamics of electricity spot prices. We also discuss the pricing of European call and put options written on electricity forward contracts.
\end{abstract}

\section{INTRODUCTION}

The aim of this paper is to propose a model for the electricity spot price dynamics. We suggest a model that, while capturing the essential characteristics of spot prices to a reasonable degree, is simple enough to yield closed form expressions for electricity forward and futures contracts and other derivatives. The standard approach in the literature is to model the logarithmic electricity spot prices through a mean-reverting process (see e.g., Lucia and Schwartz [11] and Geman and Roncoroni [9]), such that in the classical Gaussian setting the spot price dynamics becomes lognormal. For such models it is notoriously difficult to derive manageable analytical expressions for the corresponding forward and futures contracts. We propose instead to model the spot price dynamics directly by an Ornstein-Uhlenbeck process which, however, is non-Gaussian. More precisely, we consider a sum of Ornstein-Uhlenbeck processes, each of which is reverting to a mean at a different speed and having pure jump processes with only positive jumps as sources of randomness. Our proposed spot price dynamics has thus an additive structure (in contrast to exponential structure) and gives positive prices. We remark that our proposed model is motivated from a stochastic volatility model proposed by Barndorff-Nielsen and Shephard [1] in a different context.

The approaches to model prices on electricity markets can mainly be divided into two categories: spot price models and models for futures prices. A model in the latter category

Date: May 23, 2005.

Key words and phrases. Electricity markets, spot price modeling, forward and futures pricing, additive processes, Ornstein-Uhlenbeck processes. 
describes directly futures prices instead of modeling spot prices from which futures prices then are derived. The advantage of such models is that the market can be assumed to be complete and the usual risk neutral pricing machinery may be employed. Recent approaches are to transfer concepts from the Heath-Jarrow-Morton theory for interest rates to electricity markets to model the complete futures price curve (see Bjerksund et al. [5] and Benth and Koekebakker [3]). The problem with these approaches is that due to the nonstorability of electricity one cannot use arbitrage arguments to derive information about spot prices from the analysis of futures price models. Also, since futures and forwards typically have delivery periods of fully months, quarters or years, futures curve moves are much less volatile than changes in spot prices.

However, many derivatives on electricity prices depend heavily on hourly or daily prices which makes the modeling of spot prices necessary. Models in this category try to fit the dynamics of hourly (most often day ahead) prices on the spot market which then can be used to derive prices of futures and more complicated derivatives. But since no-arbitrage relations between spot and futures prices do not exist, additional assumptions have to be made in order to price derivatives based on spot price models. More specifically, one has to identify the market price of risk of the including risk factors which then yield an equivalent martingale pricing measure. It seems to be reaonable to assume at least two risk factors in spot price models, one responsible for short term hourly behaviour with strong volatility and one for more long term behaviour observed on futures markets.

There are several key characteristics of electricity spot prices that can be observed more or less distinctly in all electricity markets. We recall the three most important ones. First, electricity prices typically show very sharp spikes. This is due to inelastic demand combined with an exponentially increasing curve of marginal costs. In times of sudden changes of demand or supply (for example caused by weather conditions) this results in strong jumps of electricity prices. However, after sudden changes another feature of electricity prices is that they rather rapidly tend to revert back to a mean level, which makes a mean reverting process appropriate to model spot prices. Finally, all involved magnitudes such as the mean level, jump intensities and jump sizes exhibit seasonal behaviour over one day, week, and year. As an illustrative example we present in Figure 1 the development of the daily spot prices at Nordpool, the Nordic Electricity Exchange, spanning over the period of approximately 3 years starting in April 1997.

The paper is organized as follows: In the next section we define the spot price model, and analyze some of its properties. Section 3 calculates electricity forward and futures prices based on the proposed spot price dynamics. Expressions for the fair premium of call and put options written on these electricity forwards and futures are analyzed in Section 4. Finally, in Section 5 we conclude.

\section{Electricity Spot Price Process}

Let $\left(\Omega, P, \mathcal{F},\left\{\mathcal{F}_{t}\right\}_{t \in[0, T]}\right)$ be a complete filtered probability space, with $T<\infty$ a fixed time horizon. If $S(t)$ denotes the spot price of electricity at time $t$, then we set

$$
S(t)=\mu(t)+X(t) .
$$




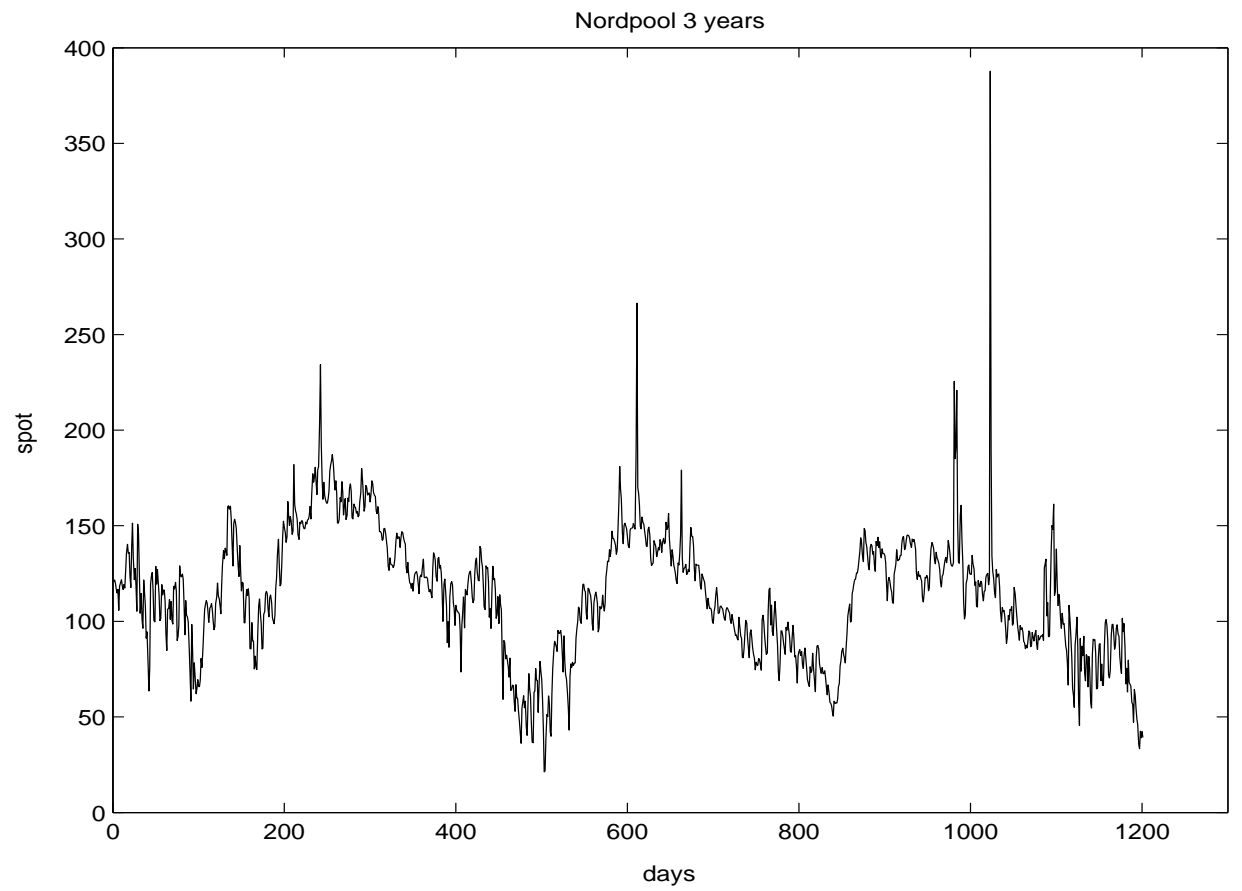

Figure 1. The daily spot price on Nordpool spanning from April 1, 1997 until July 14, 2000

Here $\mu(t)$ is a deterministic, periodic function and the stochastic process $X(t)$ is described by the following dynamics:

$$
X(t)=\sum_{i=1}^{n} w_{i} Y_{i}(t)
$$

where

$$
d Y_{i}(t)=-\lambda_{i} Y_{i}(t) d t+\sigma_{i}(t) d L_{i}(t), \quad Y_{i}(0)=y_{i}, i=1, \ldots, n,
$$

and $w_{i}$ are positive weight functions such that $\sum_{i=1}^{n} w_{i}=1, \lambda_{i}$ are positive constants and $\sigma_{i}(t)$ are positive bounded functions. The processes $L_{i}(t), i=1, \ldots, n$ are assumed to be independent increasing càdlàg pure jump processes that can be represented in terms of their jump measures $N_{i}(d t, d z), i=1, \ldots, n$ :

$$
L_{i}(t)=\int_{0}^{t} \int_{0}^{\infty} z N_{i}(d s, d z) .
$$

We suppose $L_{i}(t)$ to be integrable and that the jump measures $N_{i}(d t, d z)$ have deterministic predictable compensators $\nu_{i}(d t, d z)$ for all $i=1, \ldots, n$. These processes are also referred to as additive or Sato processes and they have independent but not necessarily stationary increments. Setting

$$
\widetilde{N}_{i}(d s, d z)=N_{i}(d s, d z)-\nu_{i}(d s, d z)
$$


we denote by $\widetilde{L}_{i}$ the compensated jump process

$$
\widetilde{L}_{i}(t)=\int_{0}^{t} \int_{0}^{\infty} z \widetilde{N}_{i}(d t, d z) .
$$

In our model we will only deal with compensators of the form

$$
\nu_{i}(d t, d z)=\rho_{i}(t) d t \nu_{i}(d z)
$$

where $\rho_{i}(t)$ is a deterministic function. The cumulant function is defined as

$$
\psi_{t}^{i}(\theta)=\int_{0}^{t} \int_{0}^{\infty}\left\{\mathrm{e}^{\mathrm{i} \theta z}-1\right\} \nu_{i}(d s, d z)
$$

where $\theta \in \mathbb{R}$.

An explicit representation of the electricity spot price is

$$
S(t)=\mu(t)+\sum_{i=1}^{n} w_{i}\left(y_{i} \mathrm{e}^{-\lambda_{i} t}+\int_{0}^{t} \sigma_{i}(u) \mathrm{e}^{-\lambda_{i}(t-u)} d L_{i}(u)\right) .
$$

We see that $S(t)$ is mean reverting to the periodic function $\mu(t)$ to which each component $Y_{i}(t)$ contributes with a speed given by $w_{i}$ and $\lambda_{i}$. The processes $L_{i}$ control the price variation (including both the daily volatile variation and the price spikes) where $\sigma_{i}(t)$ controls the seasonal variation of the jump sizes while $\rho_{i}(t)$ controls the seasonal variation of the jump intensity. Positivity of spot prices is guaranteed because the processes $L_{i}$ are increasing.

Note the periodic function $\mu(t)$ is not the mean level of the spot price but is related to this in the following way. The mean level of the spot price, let us denote it by $\theta(t)$, is usually identified by calibrating a periodic function (eventually including a trend line) to spot prices after having taken out spikes of sudden bigger price variations. Let us assume in our model, the processes $Y_{i}(t), i=1, . ., l$ are responsible for modelling the daily volatile variation while the processes $Y_{i}(t), i=l+1, . ., n$ are modelling the price spikes. Then it makes sense to require the average spot price exluding the spikes to be the mean level $\theta(t)$ :

$$
E\left[\mu(t)+\sum_{i=1}^{l} w_{i} Y_{i}(t)\right]=\theta(t)
$$

Further, in order to model the daily volatility it may seem reasonable to assume the jump intensity $\rho_{i}(t)$ and jump size $\sigma_{i}(t)$ to be constant over time. In this case we can set $\rho_{i}(t)=1$ and $L_{i}(t)$ becomes a subordinator (i.e. increasing Lévy process) for $i=1, . ., l$. It is then known that $Y_{i}(t), i=1, . ., l$, become stationary processes given the right starting point $Y_{i}(0)$ (see e.g. [1]). Moreover, starting at a deterministic value $y_{i}$ the processes $Y_{i}(t)$ are converging to the corresponding stationary processes as time goes by. If we denote the first moments of these stationary processes by $\beta_{i}:=\frac{\sigma_{i}}{\lambda_{i}} \int z \nu_{i}(d z), i=1, . ., l$, we determine the periodic function $\mu(t)$ in this case by the relation

$$
\mu(t)=\theta(t)-\sum_{i=1}^{l} w_{i} \beta_{i} .
$$

Finally, we assume that the risk-free interest rate in the market is $r>0$, which we will understand as the return from a zero-coupon bond investment. 
Example 2.1. It is not the purpose of this paper to do precise statistical analysis or calibration. However, in order to make a first assessment of the model we simulate a path of the spot dynamics using Ornstein-Uhlenbeck processes described by the following specifications: according to the time horizon considered in Fig. 1

\begin{tabular}{l|cccc} 
& $\boldsymbol{\lambda}$ & $\boldsymbol{\sigma}(\mathbf{t})$ & $\boldsymbol{\nu}(\mathbf{d z})$ & $\boldsymbol{\rho}(\mathbf{t})$ \\
\hline $\mathbf{O U}_{1}$ & 0.06 & 1 & $\Gamma(0.7 ; 60)$ & 1 \\
$\mathbf{O U}_{2}$ & 0.5 & 1 & $\Gamma(1 ; 14)$ & 10 \\
$\mathbf{O U}_{3}$ & 1.7 & 1 & $\Gamma(1 ; 280)$ & $0.05\left(\frac{2}{\left|\sin \left(\frac{\pi(t-91)}{365}\right)\right|+1}-1\right)$
\end{tabular}

Here $\Gamma(\alpha ; \gamma)$ denotes the Gamma distribution with parameters $\alpha$ and $\gamma$. The first two Ornstein-Uhlenbeck processes are responsible for the volatile variation around $\mu(t)$ and the third one models the spikes preferably occuring in winter. For $\mu(t)$ we have taken a sinus function with yearly period. The path of the spot price is simulated over a time horizon equal to the one we considered in Fig. 1 for the Nordpool spot prices. As we can see in Fig. 2, the simulated path seems to capture well, at least visually, the essential features exhibited by the Nordpool spot price sample path, like e.g. seasonality and distinct price spikes in the winter.

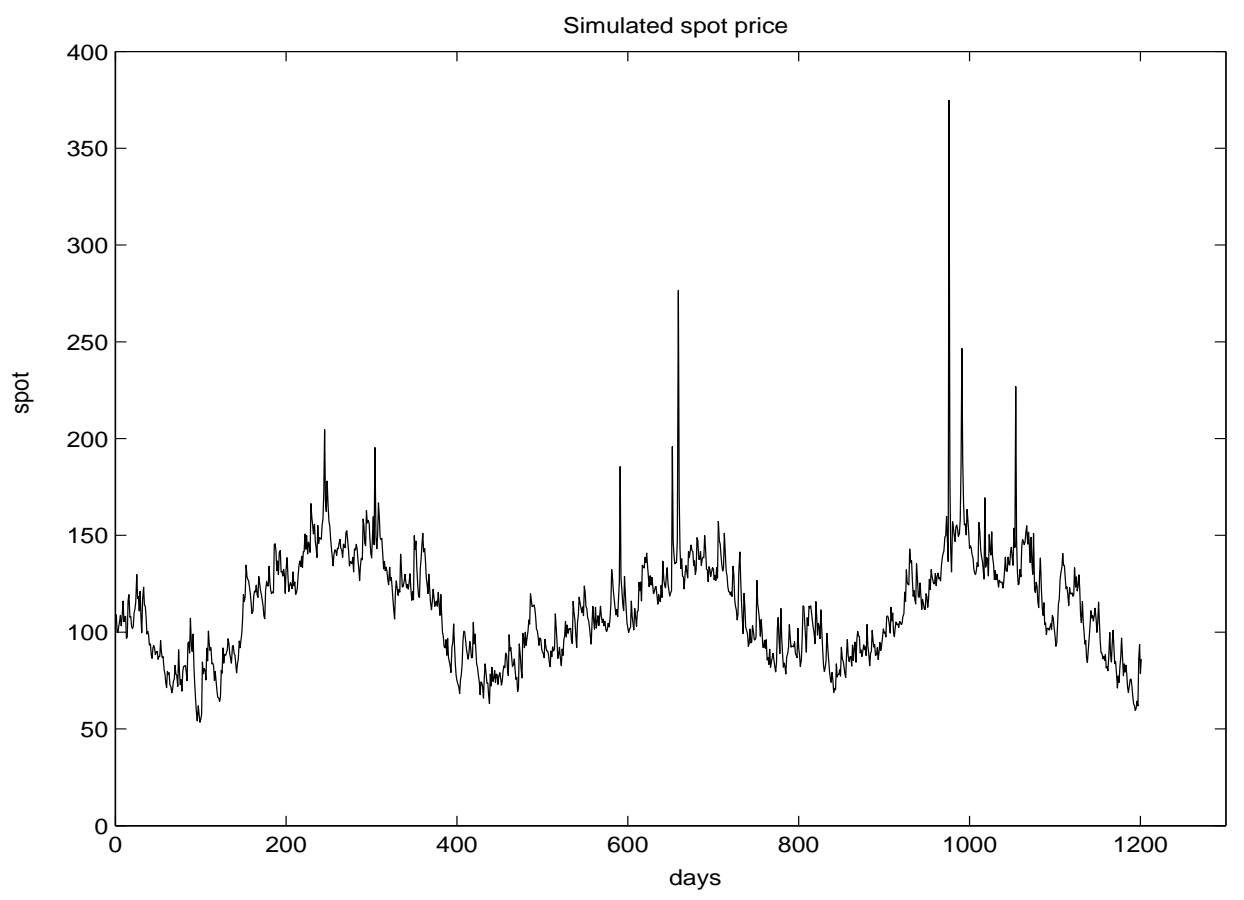

FIGURE 2. Simulation of a spot price path using the specifications given in Example 2.1.

\section{Pricing of Forwards and Futures in the Electricity Market}

In the Nordpool power market (and other power exchanges around the world) there is trade in forwards and futures contracts based on electricity. The main distinction of such 
contracts compared to other commodity markets is that electricity forwards and futures delivers the underlying commodity over a period, rather than at a fixed time. In the financial power market, these products are cash settled measured against the spot price in the settlement period.

Consider a forward contract which delivers electricity over the period $\left[T_{1}, T_{2}\right]$, where $T_{1}<T_{2} \leq T$. The electricity is delivered as a flow of rate $S(t) / T_{2}-T_{1}$ in the settlement period, giving a total delivery of

$$
\int_{T_{1}}^{T_{2}} S(u) d u /\left(T_{2}-T_{1}\right) .
$$

The contracts at Nordpool is settled financially, in the sense that the holder of the contract receives the money equivalent of this delivery. Using the arbitrage-free pricing mechanism and assuming that settlement takes place at the end of the delivery period, the electricity forward has the price $F\left(t, T_{1}, T_{2}\right)$ at time $0 \leq t \leq T_{1}$ given as

$$
F\left(t ; T_{1}, T_{2}\right)=\mathbb{E}_{Q}\left[\frac{1}{T_{1}-T_{1}} \int_{T_{1}}^{T_{2}} S(u) d u \mid \mathcal{F}_{t}\right],
$$

where $Q$ is an equivalent martingale measure. Since there is no tradable underlying, all equivalent measures $Q \sim P$ will become martingale measures. Thus, to derive electricity forward prices we need to identify the market price of the involved risk factors in order to choose the pricing measure $Q$.

Note in passing that the forward price coincides with the price of the correponding futures contract when the risk-free interest rate $r$ is constant, as we assume in this paper. Furthermore, after interchanging expectation with integration with respect to time, it holds that

$$
F\left(t ; T_{1}, T_{2}\right)=\frac{1}{T_{2}-T_{1}} \int_{T_{1}}^{T_{2}} f(u, \tau) d \tau
$$

where

$$
f(t, \tau)=\mathbb{E}_{Q}\left[S(\tau) \mid \mathcal{F}_{t}\right] .
$$

Note that $f(t, \tau)$ is the price of a forward contract at time $t$ delivering electricity at the fixed time $\tau \geq t$. Thus, the electricity forwards can be considered as a continuous stream of forward contracts with fixed delivery times over the delivery period. Due to the simple structure of our model we may use this to derive a very tractable dynamics of the electricity forwards, the task we now turn our attention to.

To specify a class of pricing measures, we consider equivalent martingale measures $Q$ which are characterized through Radon-Nikodym derivatives of the form

$$
\frac{d Q}{d P}=\prod_{i=1}^{n} \mathcal{E}\left(M^{i}\right),
$$

where

$$
M_{t}^{i}=\int_{0}^{t} \int_{\mathbb{R}_{0}}\left(\phi_{i}(z, t)-1\right) \tilde{N}_{i}(d z, d t), \quad 0 \leq t \leq T
$$

for some positive deterministic integrand $\phi_{i}(z, t)$. Then $M^{i}$ and the corresponding DoleansDade exponential $\mathcal{E}\left(M^{i}\right)$ are actually martingales (see Lemmata 4.2 and 4.4 in [10] which 
also hold in this case), and the Girsanov theorem for random measures yields that the jump measure $N_{i}(d z, d t)$ has the compensation $\phi_{i}(z, t) \nu_{i}(d z, d t)$ under $Q$. Let

$$
\hat{\nu}_{i}(d z, d t):=\phi_{i}(z, t) \nu_{i}(d z, d t) .
$$

We have the following result:

Proposition 3.1. The price of an electricity forward $F\left(t, T_{1}, T_{2}\right)$ at time $t$ and delivery period $\left[T_{1}, T_{2}\right], t \leq T_{1}$, is given as

$$
F\left(t ; T_{1}, T_{2}\right)=F\left(0 ; T_{1}, T_{2}\right)+\sum_{i=1}^{n} \frac{w_{i}}{\lambda_{i}\left(T_{2}-T_{1}\right)} \int_{0}^{t} \sigma_{i}(s)\left(e^{-\lambda_{i}\left(T_{1}-s\right)}-e^{-\lambda_{i}\left(T_{2}-s\right)}\right) d \bar{L}_{i}(s)
$$

where

$$
\begin{aligned}
F\left(0 ; T_{1}, T_{2}\right)=\frac{1}{T_{2}-T_{1}} \int_{T_{1}}^{T_{2}}\{\mu(u)+ & \\
+ & \left.\sum_{i=1}^{n} w_{i}\left(y_{i} e^{-\lambda_{i} u}+\int_{0}^{u} \int_{\mathbb{R}^{+}} \sigma_{i}(s) e^{-\lambda_{i}(u-s)} z \hat{\nu}_{i}(d z, d s)\right)\right\} d u
\end{aligned}
$$

and $\bar{L}_{i}(t)$ is the compensated jump process under $Q$, i.e.

$$
\bar{L}_{i}(t)=\int_{0}^{t} \int_{\mathbb{R}^{+}} z\left\{N(d z, d s)-\hat{\nu}_{i}(d z, d s)\right\} .
$$

Proof. The argument goes by a straightforward calculation, using the independent increment property of $L_{i}$ under $Q$

$$
\begin{aligned}
F & \left(t ; T_{1}, T_{2}\right) \\
= & \frac{1}{T_{2}-T_{1}} \int_{T_{1}}^{T_{2}} \mathbb{E}_{Q}\left[S(u) \mid \mathcal{F}_{t}\right] d u \\
= & \frac{1}{T_{2}-T_{1}} \int_{T_{1}}^{T_{2}}\left\{\mu(u)+\sum_{i=1}^{n} w_{i} y_{i} \mathrm{e}^{-\lambda_{i} u}\right\} d u \\
& +\sum_{i=1}^{n} \frac{w_{i}}{T_{2}-T_{1}} \int_{T_{1}}^{T_{2}} \mathbb{E}_{Q}\left[\int_{0}^{u} \sigma_{i}(s) \mathrm{e}^{-\lambda_{i}(u-s)} d L_{i}(s) \mid \mathcal{F}_{t}\right] d u \\
= & \frac{1}{T_{2}-T_{1}} \int_{T_{1}}^{T_{2}}\left\{\mu(u)+\sum_{i=1}^{n} w_{i}\left(y_{i} \mathrm{e}^{-\lambda_{i} u}+\int_{0}^{u} \int_{\mathbb{R}^{+}} \sigma_{i}(s) \mathrm{e}^{-\lambda_{i}(u-s)} z \hat{\nu}_{i}(d z, d s)\right)\right\} d u \\
& +\sum_{i=1}^{n} \frac{w_{i}}{T_{2}-T_{1}} \int_{T_{1}}^{T_{2}} \mathbb{E}_{Q}\left[\int_{0}^{u} \sigma_{i}(s) \mathrm{e}^{-\lambda_{i}(u-s)} d \bar{L}_{i}(s) \mid \mathcal{F}_{t}\right] d u \\
= & \frac{1}{T_{2}-T_{1}} \int_{T_{1}}^{T_{2}}\left\{\mu(u)+\sum_{i=1}^{n} w_{i}\left(y_{i} \mathrm{e}^{-\lambda_{i} u}+\int_{0}^{u} \int_{\mathbb{R}^{+}} \sigma_{i}(s) \mathrm{e}^{-\lambda_{i}(u-s)} z \hat{\nu}_{i}(d z, d s)\right)\right\} d u \\
& +\sum_{i=1}^{n} \frac{w_{i}}{T_{2}-T_{1}} \int_{T_{1}}^{T_{2}} \int_{0}^{t} \sigma_{i}(s) \mathrm{e}^{-\lambda_{i}(u-s)} d \bar{L}_{i}(s) d u
\end{aligned}
$$




$$
=F\left(0 ; T_{1}, T_{2}\right)+\sum_{i=1}^{n} \frac{w_{i}}{-\lambda_{i}\left(T_{2}-T_{1}\right)} \int_{0}^{t} \sigma_{i}(s)\left(\mathrm{e}^{-\lambda_{i}\left(T_{2}-s\right)}-\mathrm{e}^{-\lambda_{i}\left(T_{1}-s\right)}\right) d \bar{L}_{i}(s)
$$

This concludes the proof.

In particular, if the $L_{i}(t)$ 's were subordinators (i.e. increasing Lévy processes) such that under $Q$ the processes $L_{i}(t)$ were again subordinators with $E_{Q}\left[L_{i}(1)\right]=\hat{\pi}_{i}$, we would get that $\bar{L}_{i}(t)$ is a compensated subordinator under $Q$ and

$$
F\left(0 ; T_{1}, T_{2}\right)=\frac{1}{T_{2}-T_{1}} \int_{T_{1}}^{T_{2}}\left\{\mu(u)+\sum_{i=1}^{n} w_{i}\left(y_{i} \mathrm{e}^{-\lambda_{i} u}+\hat{\pi}_{i} \int_{0}^{u} \sigma_{i}(s) \mathrm{e}^{-\lambda_{i}(u-s)} d s\right)\right\} d u .
$$

It may be desirable to represent the electricity forward price in terms of the spot price. Since in our model we have represented the spot price essentially as the sum of OrnsteinUhlenbeck processes, we are able to derive a representation of the forward price in terms of these only. The next proposition states the exact result:

Proposition 3.2. The price of an electricity forward $F\left(t, T_{1}, T_{2}\right)$ at time $t$ and delivery period $\left[T_{1}, T_{2}\right], t \leq T_{1}$, is given as

$$
\begin{aligned}
F\left(t ; T_{1}, T_{2}\right)= & \frac{1}{T_{2}-T_{1}} \int_{T_{1}}^{T_{2}}\left(\mu(u)+\sum_{i=1}^{n} w_{i} \int_{t}^{u} \int_{\mathbb{R}^{+}} \sigma_{i}(s) e^{-\lambda_{i}(u-s)} z \hat{\nu}_{i}(d z, d s)\right) d u \\
& +\sum_{i=1}^{n} \frac{w_{i} Y_{i}(t)}{\lambda_{i}\left(T_{2}-T_{1}\right)}\left(e^{-\lambda_{i}\left(T_{1}-t\right)}-e^{-\lambda_{i}\left(T_{2}-t\right)}\right) .
\end{aligned}
$$

Proof. Observe that

$$
X(u)=\sum_{i=1}^{n} w_{i}\left(Y_{i}(t) \mathrm{e}^{-\lambda_{i}(u-t)}+\int_{t}^{u} \sigma_{i}(s) \mathrm{e}^{-\lambda_{i}(u-s)} d L_{i}(s)\right) .
$$

Thus, we can calculate as follows to derive the desired result:

$$
\begin{aligned}
F\left(t ; T_{1}, T_{2}\right) & \frac{1}{T_{2}-T_{1}} \int_{T_{1}}^{T_{2}} \mathbb{E}_{Q}\left[S(u) \mid \mathcal{F}_{t}\right] d u \\
= & \frac{1}{T_{2}-T_{1}} \int_{T_{1}}^{T_{2}}\{\mu(u) \\
& \left.+\sum_{i=1}^{n} w_{i}\left(Y_{i}(t) \mathrm{e}^{-\lambda_{i}(u-t)}+\mathbb{E}_{Q}\left[\int_{t}^{u} \sigma_{i}(s) \mathrm{e}^{-\lambda_{i}(u-s)} d L_{i}(s) \mid \mathcal{F}_{t}\right]\right)\right\} d u \\
= & \frac{1}{T_{2}-T_{1}} \int_{T_{1}}^{T_{2}}\left\{\mu(u)+\sum_{i=1}^{n} w_{i}\left(Y_{i}(t) \mathrm{e}^{-\lambda_{i}(u-t)}+\int_{t}^{u} \int_{\mathbb{R}^{+}} \sigma_{i}(s) \mathrm{e}^{-\lambda_{i}(u-s)} z \hat{\nu}_{i}(d z, d s)\right.\right. \\
& \left.\left.+\mathbb{E}_{Q}\left[\int_{t}^{u} \sigma_{i}(s) \mathrm{e}^{-\lambda_{i}(u-s)} d \bar{L}_{i}(s) \mid \mathcal{F}_{t}\right]\right)\right\} d u \\
= & \frac{1}{T_{2}-T_{1}} \int_{T_{1}}^{T_{2}}\{\mu(u)
\end{aligned}
$$




$$
\begin{aligned}
& \left.+\sum_{i=1}^{n} w_{i}\left(Y_{i}(t) \mathrm{e}^{-\lambda_{i}(u-t)}+\int_{t}^{u} \int_{\mathbb{R}^{+}} \sigma_{i}(s) \mathrm{e}^{-\lambda_{i}(u-s)} z \hat{\nu}_{i}(d z, d s)\right)\right\} d u \\
= & \frac{1}{T_{2}-T_{1}} \int_{T_{1}}^{T_{2}}\left(\mu(u)+\sum_{i=1}^{n} w_{i} \int_{t}^{u} \int_{\mathbb{R}^{+}} \sigma_{i}(s) \mathrm{e}^{-\lambda_{i}(u-s)} z \hat{\nu}_{i}(d z, d s)\right) d u \\
& -\sum_{i=1}^{n} \frac{w_{i} Y_{i}(t)}{\lambda_{i}\left(T_{2}-T_{1}\right)}\left(\mathrm{e}^{-\lambda_{i}\left(T_{2}-t\right)}-\mathrm{e}^{-\lambda_{i}\left(T_{1}-t\right)}\right) .
\end{aligned}
$$

This concludes our proof.

Remark that the explicit form of the forward dynamics can be used to calibrate the model to observations. Rather than first estimating the parameters in the spot dynamics, and next deriving forward prices, we can start out with the forward dynamics in Prop. 3.2 as the model, and estimate the parameters using forward observations. From this point of view, one can say that we use a Heath-Jarrow-Morton approach taken from interest rate theory to model the forward dynamics, with the advantage that there is an underlying spot dynamics connected to this. ${ }^{1}$ Note that since the data is observed under the probability $P$, one needs to consider the objective dynamics, and not the risk-neutral as stated in the proposition.

In the Nordpool market there exist forward contracts where the delivery period is overlapping. One can for example trade in contracts with yearly delivery, but at the same time the market also offers contracts with delivery in each quarters of the year. In theory, such contracts must satisfy the no-arbitrage condition

$$
F\left(t ; T_{1}, T_{2}\right)=\sum_{i=1}^{n-1} \frac{\tau_{i+1}-\tau_{i}}{T_{2}-T_{1}} F\left(t ; \tau_{i}, \tau_{i+1}\right)
$$

where $T_{1}=\tau_{1}<\tau_{2}<\ldots<\tau_{n}=T_{2}$. This is indeed the case with our model.

In order to illustrate the advantage of our additive model compared to exponential models, we calculate the dynamics of a swap in the corresponding exponential model. For now, assume that $S(t)$ is given by

$$
S(t)=\exp (\mu(t)+X(t))
$$

where $X(t)$ is as before (but not necessary restricted to have only positive jumps). Then the swap dynamic can be calculated as follows.

Proposition 3.3. Suppose that the spot dynamics is given by (3.3), and that $S(t)$ is integrable. Then the electricity forward price $F\left(t ; T_{1}, T_{2}\right)$ at time $t$ with delivery over the period $\left[T_{1}, T_{2}\right]$ is given by

$$
F\left(t ; T_{1}, T_{2}\right)=\frac{1}{T_{1}-T_{1}} \int_{T_{1}}^{T_{2}} G(u) \prod_{i=1}^{n} M_{i}(t ; u) d u
$$

\footnotetext{
${ }^{1}$ If one starts out with a specification of the forward dynamics directly, it is not always the case that one can associate a spot dynamics. See Benth and Koekebakker [3] for more on this.
} 
where

$$
G(u)=\exp \left\{\mu(u)+\sum_{i=1}^{n} w_{i}\left(y_{i} e^{-\lambda_{i} u}+\int_{0}^{u} \int_{\mathbb{R}^{+}}\left(e^{z w_{i} \sigma_{i}(r) e^{-\lambda_{i}(u-r)}}-1\right) \hat{\nu}_{i}(d z, d r)\right)\right\}
$$

and $M_{i}(t ; u)$ is given by

$$
d M_{i}(t ; u)=M_{i}(t ; u)\left(e^{z w_{i} \sigma_{i}(r) e^{-\lambda_{i}(u-r)}}-1\right)\left\{N_{i}(d z, d t)-\hat{\nu}_{i}(d z, d s)\right\} .
$$

Proof. The argument goes by a straightforward calculation.

$$
\begin{aligned}
& F\left(t ; T_{1}, T_{2}\right) \\
& =\frac{1}{T_{2}-T_{1}} \int_{T_{1}}^{T_{2}} \mathbb{E}_{Q}\left[S(u) \mid \mathcal{F}_{t}\right] d u \\
& =\frac{1}{T_{2}-T_{1}} \int_{T_{1}}^{T_{2}}\left\{\mathbb{E}_{Q}[\exp (\mu(u)\right. \\
& \left.\left.\left.+\sum_{i=1}^{n} w_{i}\left(y_{i} \mathrm{e}^{-\lambda_{i} u}+\int_{0}^{u} \sigma_{i}(s) \mathrm{e}^{-\lambda_{i}(u-s)} d L_{i}(s)\right)\right) \mid \mathcal{F}_{t}\right]\right\} d u \\
& =\frac{1}{T_{2}-T_{1}} \int_{T_{1}}^{T_{2}}\{\exp (\mu(u) \\
& \left.+\sum_{i=1}^{n} w_{i} y_{i} \mathrm{e}^{-\lambda_{i} u}+\int_{0}^{u} \int_{\mathbb{R}^{+}}\left(\mathrm{e}^{z w_{i} \sigma_{i}(s) \mathrm{e}^{-\lambda_{i}(u-s)}}-1\right) \hat{\nu}_{i}(d z, d s)\right) \\
& \mathbb{E}_{Q}\left[\operatorname { e x p } \left(\sum_{i=1}^{n} w_{i} \int_{0}^{u} \sigma_{i}(s) \mathrm{e}^{-\lambda_{i}(u-s)} d L_{i}(s)\right.\right. \\
& \left.\left.\left.-\int_{0}^{u} \int_{\mathbb{R}^{+}}\left(\mathrm{e}^{z w_{i} \sigma_{i}(s) \mathrm{e}^{-\lambda_{i}(u-s)}}-1\right) \hat{\nu}_{i}(d z, d s)\right) \mid \mathcal{F}_{t}\right]\right\} d u \\
& =\frac{1}{T_{2}-T_{1}} \int_{T_{1}}^{T_{2}}\{\exp (\mu(u) \\
& \left.+\sum_{i=1}^{n} w_{i} y_{i} \mathrm{e}^{-\lambda_{i} u}+\int_{0}^{u} \int_{\mathbb{R}^{+}}\left(\mathrm{e}^{z w_{i} \sigma_{i}(s) \mathrm{e}^{-\lambda_{i}(u-s)}}-1\right) \hat{\nu}_{i}(d z, d s)\right) \\
& \exp \left(\sum_{i=1}^{n} w_{i} \int_{0}^{t} \sigma_{i}(s) \mathrm{e}^{-\lambda_{i}(u-s)} d L_{i}(s)\right. \\
& \left.\left.-\int_{0}^{t} \int_{\mathbb{R}^{+}}\left(\mathrm{e}^{z w_{i} \sigma_{i}(s) \mathrm{e}^{-\lambda_{i}(u-s)}}-1\right) \hat{\nu}_{i}(d z, d s)\right)\right\} d u \\
& =\frac{1}{T_{2}-T_{1}} \int_{T_{1}}^{T_{2}} G(u) \prod_{i=1}^{n} M_{i}(t ; u) d u
\end{aligned}
$$

This concludes the calculation. 
Note in (3.4) the integral expression for which there is no closed form expression in general. This is the main drawback with the exponential models, which, furthermore, lead to quite complicated expressions for the price of call and put options. The additive model, on the other hand, lends itself to an analysis of option prices in a rather neat way.

The reader should notice that the proof in the proposition above assumes that the jump process $L_{i}$ is of finite variation. We can easily extend the result to general jump processes with infinite variation, however, the main message is not altered. The electricity forward prices do not allow for any explicit pricing mechanism when the spot is based on an exponential model.

\section{Pricing of Options on Electricity Forwards and Futures}

The Nordpool electricity market organizes standardized trading in European call and put options written of electricity forwards and futures. We analyze the pricing of these based on the choice of risk-neutral measure $Q$ made in the section above. By employing the method of Fourier transform along with the cumulant functions of the jump processes involved, we can derive expression for the price that at least lend themselves to numerical pricing by fast Fourier transform techniques.

Before setting off, let us introduce the following notation: Define $\Sigma_{i}\left(t, T_{1}, T_{2}\right)$ as

$$
\Sigma_{i}\left(t, T_{1}, T_{2}\right)=\frac{w_{i} \sigma_{i}(t)}{\lambda_{i}\left(T_{2}-T_{1}\right)}\left(\mathrm{e}^{-\lambda_{i}\left(T_{1}-t\right)}-\mathrm{e}^{-\lambda_{i}\left(T_{2}-t\right)}\right) .
$$

Then the forward dynamics can be written

$$
F\left(t ; T_{1}, T_{2}\right)=F\left(0 ; T_{1}, T_{2}\right)+\sum_{i=1}^{n} \int_{0}^{t} \Sigma_{i}\left(u ; T_{1}, T_{2}\right) d \bar{L}_{i}(u) .
$$

We also introduce the notation $\widetilde{\psi}_{t, T}^{i}(\theta)$ to denote a sort of cumulant of $L_{i}$ with respect to the measure $Q$, more specifically

$$
\widetilde{\psi}_{t, T}^{i}(\theta):=\ln \mathbb{E}_{Q}\left[\exp \left(\mathrm{i} \int_{t}^{T} \theta(s) d L_{i}(s)\right)\right]=\int_{t}^{T} \int_{0}^{\infty}\left\{\mathrm{e}^{\mathrm{i} \theta(s) z}-1\right\} \widehat{\nu}_{i}(d z, d s)
$$

for deteministic functions $\theta(s)$. Let $K$ be the strike price at time $T$, where $T \leq T_{1}$, the start of the delivery period of the underlying electricity forward. The price of a put option contract at time $t \leq T$ written on a forward with delivery period $\left[T_{1}, T_{2}\right]$ is given by the expectation

$$
p\left(t ; T ; T_{1}, T_{2}\right)=\mathrm{e}^{-r(T-t)} \mathbb{E}_{Q}\left[\max \left(K-F\left(T ; T_{1}, T_{2}\right), 0\right) \mid \mathcal{F}_{t}\right] .
$$

Observe that the payoff function for a put option $g(x)=\max (K-x, 0)$ is in $L^{1}([0, \infty))$. Moreover, by extending the function naturally to be zero on the negative half of the real line, we have a payoff function which belongs to the space $L^{1}(\mathbb{R})$, and thus the Fourier transform of it can be defined. Let us more generally consider payoff functions $g \in L^{1}(\mathbb{R})$, and the corresponding option price which becomes

$$
p\left(t ; T ; T_{1}, T_{2}\right)=\mathrm{e}^{-r(T-t)} \mathbb{E}_{Q}\left[g\left(F\left(T ; T_{1}, T_{2}\right)\right) \mid \mathcal{F}_{t}\right] .
$$

We have the following result: 
Proposition 4.1. If $g\left(F\left(T, T_{1}, T_{2}\right)\right) \in L^{1}(Q)$, then we have that

$$
p\left(t ; T ; T_{1}, T_{2}\right)=e^{-r(T-t)}\left(g \star \Phi_{t, T}\right)\left(F\left(t ; T_{1}, T_{2}\right)\right)
$$

where the function $\Phi_{t, T}$ is defined via its Fourier transform

$$
\widehat{\Phi}_{t, T}(y)=\exp \left(\sum_{i=1}^{n} \widetilde{\psi}_{t, T}^{i}\left(y \Sigma_{i}\left(\cdot, T_{1}, T_{2}\right)\right)\right),
$$

and $\star$ is the convolution product.

Proof. The proof goes via the use of the Fourier transform. Recall first that

$$
g(x)=\frac{1}{2 \pi} \int \widehat{g}(y) \mathrm{e}^{\mathrm{i} y x} d y
$$

where $\widehat{g}$ is the Fourier transform of $g$. Thus, we have

$$
\begin{aligned}
& \mathbb{E}_{Q}\left[g\left(F\left(T ; T_{1}, T_{2}\right)\right) \mid \mathcal{F}_{t}\right] \\
& =\frac{1}{2 \pi} \int \widehat{g}(y) \mathbb{E}_{Q}\left[\exp \left(\mathrm{i} y F\left(T ; T_{1}, T_{2}\right)\right) \mid \mathcal{F}_{t}\right] d y \\
& =\frac{1}{2 \pi} \int \widehat{g}(y) \exp \left(\mathrm{i} y F\left(0 ; T_{1}, T_{2}\right)\right) \times \mathbb{E}_{Q}\left[\exp \left(\mathrm{i} y \sum_{i=1}^{n} \int_{0}^{T} \Sigma_{i}\left(s ; T_{1}, T_{2}\right) d \bar{L}_{i}(s)\right) \mid \mathcal{F}_{t}\right] d y \\
& =\frac{1}{2 \pi} \int \widehat{g}(y) \exp \left(\mathrm{i} y F\left(t ; T_{1}, T_{2}\right)\right) \times \mathbb{E}_{Q}\left[\exp \left(\mathrm{i} y \sum_{i=1}^{n} \int_{t}^{T} \Sigma_{i}\left(s ; T_{1}, T_{2}\right) d \bar{L}_{i}(s)\right) \mid \mathcal{F}_{t}\right] d y .
\end{aligned}
$$

The independent increment property of $\bar{L}_{i}$ yields

$$
\begin{aligned}
& \mathbb{E}_{Q}\left[g\left(F\left(T ; T_{1}, T_{2}\right)\right) \mid \mathcal{F}_{t}\right] \\
& =\frac{1}{2 \pi} \int \widehat{g}(y) \exp \left(\mathrm{i} y F\left(t ; T_{1}, T_{2}\right)\right) \times \mathbb{E}_{Q}\left[\exp \left(\mathrm{i} y \sum_{i=1}^{n} \int_{t}^{T} \Sigma_{i}\left(s ; T_{1}, T_{2}\right) d \bar{L}_{i}(s)\right)\right] d y \\
& =\frac{1}{2 \pi} \int \widehat{g}(y) \exp \left(\mathrm{i} y F\left(t ; T_{1}, T_{2}\right)+\sum_{i=1}^{n} \widetilde{\psi}_{t, T}^{i}\left(y \Sigma_{i}\left(\cdot ; T_{1}, T_{2}\right)\right)\right) d y
\end{aligned}
$$

This proves the result.

Of course, the payoff function of a call option does not belong to the space $L^{1}(\mathbb{R})$, and a-priori we cannot use the technique above to calculate the price of a call option. Nevertheless, its price is easily obtained from the call-put parity. Alternatively, one can introduce an exponential damping of the payoff as in Carr and Madan [6]. We refer the reader to Carr and Madan [6] for the details on this.

The reader should note that it is in general not possible to work out such explicit expressions when basing the electricity forward prices on an exponential model.

\section{Conclusion}

The most common models used for spot price dynamics in electricity markets are of geometric type, and in general not feasible for calculating expression for electricity forward and futures prices. Although they may describe well the stylized facts of electricity spot prices, they become unfeasible for further analysis of derivatives pricing. We have proposed 
an additive model that meets the features of spot prices like seasonality and price spikes. The model ensures positivity of prices since the stochastic price fluctuations are modeled by employing an increasing jump process. We have demonstrated that the process is far better manageable in order to represent and price derivatives, since forward and futures prices can be calculated analytically and plain vanilla options written on these can be analyzed by Fourier techniques.

In this paper we only included a first "visual" test of our model by simulating a sample path of the spot price. This test confirmed our hope that the model is well suited to capture the stylized facts of spot prices at least to an acceptable degree. It is the purpose of future work to make a more precise statistical analysis of the quality of the model.

Acknowledgements We are grateful to Steen Koekebakker for inspiring discussions.

\section{REFERENCES}

[1] Barndorff-Nielsen, O. E., and Shephard, N. (2001): Non-Gaussian Ornstein-Uhlenbeck-based models and some of their uses in financial economics, J.R.Statist. Soc. B, 63 (part2).

[2] Benth, F.E., Ekeland, L., Hauge, R., and Nielsen, B.F. (2003). On arbitrage-free pricing of forward contracts in energy market. Appl. Math. Finance, 10(4), pp. 325-336.

[3] Benth, F.E. and Koekebakker, S. (2005). Stochastic modeling of forward and futures contracts in electricity markets. Manuscript

[4] Black, F. (1976). The pricing of commodity contracts. J. Financial Econom., 3, pp. 167-179.

[5] Bjerksund, P., Rasmussen, H. and Stensland, G. (2000). Valuation and risk management in the Nordic electricity market. Working paper, Institute of finance and management sciences, Norwegian School of Economics and Business Administration.

[6] Carr, M., Madan, D.B. (1999), Option valuation using the fast Fourier transform, J. Comp. Finance, 2(4), pp. 61-73.

[7] Clewlow, L. and Strickland, C. (2000). Energy Derivatives: Pricing and Risk Management. Lacima Publications.

[8] Eydeland, A. and Wolyniec, K. (2003). Energy and Power Risk Management. John Wiley \& Sons.

[9] Geman, H., Roncoroni, A.:Understanding the fine structure of electricity prices, working paper.

[10] Kallsen, J.: Optimal portfolios for exponential Lévy processes, Math Meth Oper Res (2000) 51, pp. 357-374.

[11] Lucia, J. and Schwartz, E. S. (2002). Electricity Prices and Power Derivatives: Evidence from the Nordic Power Exchange. Rev. Derivatives Research, 5(1), pp. 5-50.

[12] Pilipovic, D. (1988). Energy Risk. McGraw Hill.

[13] Schwartz, E. S. (1997). The stochastic behaviour of commodity prices: Implications for valuation and hedging. J. Finance, LII(3), pp. 923-973.

(Fred Espen Benth), Centre of Mathematics for Applications, University of Oslo, P.O. Box 1053, Blindern, N-0316 Oslo, Norway, And, Agder University College, School of Management, Serviceboks 422, N-4604 Kristiansand, Norway

E-mail address: fredb@math.uio.no

$U R L:$ http: //www.math.uio.no/ fredb/

(Jan Kallsen), Department of Mathematics, Munich University of Technology, D-85784 Garching (Munich), Germany

E-mail address: kallsen@ma.tum.de

(Thilo Meyer-Brandis), Center of Mathematics for Applications, University of Oslo, P.O. Box 1053, Blindern, N-0316 Oslo, Norway,

E-mail address: meyerbr@math.uio.no 